\title{
Determinantes de la estructura de capital: un estudio empírico del sector manufacturero en Ecuador
}

\author{
Determinants of capital structure: an empirical study of the manufacturing \\ sector at Ecuador
}

\author{
Herenia Gutiérrez Ponce ${ }^{1 *}$, Christian Morán Montalvo², \\ Ricardo Posas Murillo ${ }^{2}$ \\ ${ }^{1}$ Universidad Autónoma de Madrid, España \\ ${ }^{2}$ Universidad de Especialidades Espíritu Santo, Ecuador
}

Recibido el 20 de diciembre de 2017; aceptado el 21 de mayo de 2018

Disponible en Internet el: 5 de noviembre de 2018

\section{Resumen}

El objetivo de este estudio es descubrir los determinantes de la estructura de capital de 304 empresas del sector manufacturero de Guayaquil, durante el periodo 2012-2016 y qué teoría condensa mejor las decisiones de financiación de las empresas. La importancia del mismo, reside en verificar los determinantes del endeudamiento en empresas de un país en vías de desarrollo y comparar los resultados con las predicciones de las teorías sobre el Equilibrio Estático y sobre la Jerarquía Financiera. Como metodología se ha empleado una estructura de datos de panel junto a un modelo de efectos fijos. Tras la revisión de la literatura se ha utilizado un modelo de regresión de Mínimos Cuadrados Ordinarios para descubrir la correlación y significación estadística entre la variable dependiente (ratio de endeudamiento) y un conjunto de variables independientes seleccionadas. En este estudio, la teoría de la Jerarquía Financiera tuvo mayor poder explicativo que la del Equilibrio Estático. Sin embargo, no se encontró evidencia estadística que sustente la importancia de las expectativas de crecimiento sobre la estructura de capital corporativa.

Códigos JEL: G32, C12

Palabras clave: Financiamiento; estructura de capital; manufactura; endeudamiento; Equilibrio Estático; Jerarquía Financiera.

\footnotetext{
*Autor para correspondencia.

Correo electrónico: herenia.gutierrez@uam.es (H. Gutiérrez Ponce)

La revisión por pares es responsabilidad de la Universidad Nacional Autónoma de México.
} 


\begin{abstract}
The objective of this study is to discover the determinants of the capital structure of 304 companies in the manufacturing sector of Guayaquil, during the period 2012-2016 and which theory best condenses the financing decisions of companies. The importance of it lies in verifying the determinants of indebtedness in companies in a developing country and comparing the results with the predictions of the Trade-off and the Pecking Order theories. As a methodology, a panel data structure has been used together with a fixed effects model. After reviewing the literature, an Ordinary Least Squares regression model was used to discover the correlation and statistical significance between the dependent variable (debt ratio) and a set of selected independent variables. In this study, the Pecking Order theory had a greater explanatory power than the Trade-off theory. However, no statistical evidence was found to support the importance of growth expectations on the corporate capital structure.
\end{abstract}

JEL Codes: G32, C12

Keywords: Financing; capital structure; manufacturing; leverage; Trade-off; Pecking Order

\title{
Introducción
}

En los últimos años, se viene observando que uno de los principales objetivos de los administradores es el de incrementar el valor de la empresa para los accionistas. Naturalmente, una empresa requiere de activos, ya sean tangibles o intangibles, para llevar a cabo su actividad, crear valor y lograr sus objetivos empresariales. El financiamiento de estos activos puede llevarse a cabo mediante tres opciones: a) obtención de deuda (bancaria o corporativa); b) aporte de los accionistas y c) mediante fondos propios. Decidir la mezcla o combinación de deuda y de capital puede ser un ejercicio difícil pues, un endeudamiento alto podría incrementar las probabilidades de bancarrota, simultáneamente reduce el pago de impuestos y supone por ello una ventaja fiscal. Por otra parte, poca deuda implicaría una reducción del rendimiento a causa de mayor presión fiscal y conlleva a problemas de sobreinversión, seguido de una reducción del rendimiento de los accionistas.

La importancia de estudiar la estructura de capital de las empresas reside en generar modelos que puedan condensar, y predecir, los factores más relevantes que influyan en la decisión de obtener una estructura de capital "óptima" que permita hacer un uso eficiente de los recursos. Fueron Modigliani y Miller quienes en 1958 desarrollaron la primera teoría acerca de la estructura de capital, en donde indicaron que en un mercado perfecto el valor de la firma no tiene relación con su estructura de capital, y que los impuestos, el costo del capital, la asimetría de información y los costos de bancarrota tampoco afectarían, sino que su valor viene dado por el de los activos que esta poseyera (Modigliani \& Miller, 1958).

Sin embargo, el problema con las teorías de Modigliani \& Miller es que estas asumen escenarios irreales. En un trabajo reciente de (Serrasqueiro, Matias, \& Salsa, 2016), indican que las ventajas fiscales generadas por el uso de la deuda agregaban valor sustancial a las empresas, principalmente por el ahorro de pago de impuestos. También Miller (1977) en su trabajo incluyó el efecto de los impuestos en el análisis y determinó que estos solo afectan a un nivel macro y no a las empresas individualmente. DeAngelo \& Masulis (1980) indicaron que no solamente se podía reducir el pago de impuestos mediante el endeudamiento, sino que existían sustitutos (depreciación, amortización, créditos fiscales por inversión, entre otros) que no incrementaran los pasivos de las corporaciones. Por otro lado, Myers (1974) argumentó 
que las corporaciones preferirían deuda sobre capital por dos razones: 1) el costo es menor y 2) los inversionistas considerarían que la empresa estaría sobrevalorada y podrían restringir la emisión de nuevo capital.

A partir de ahí surgió una voluminosa cantidad de teorías y modelos alternativos, tales que incluyeran las imperfecciones de mercado y permitieran a los directores corporativos tomar decisiones financieras sustentadas en una base objetiva y comprobada. Varias de estas han fallado en gran parte, puesto que las condiciones económicas y financieras pueden ser muy distintas en función de la región donde se estudie, lo que conllevaría a una neutralización de la validez de tales modelos (Booth, Aivazian, Demirguc-Kunt, \& Maksimovic, 2001). Además, las teorías difieren en su énfasis y no se ha llegado a un consenso entre los académicos (Sheikh \& Wang, 2011) respecto a cuáles o cuál logra sintetizar mejor el comportamiento financiero de las firmas. Un ejemplo de ello es que investigaciones con enfoques alternativos han estudiado la relación entre las compensaciones de los administradores y la estructura de capital de las compañías que dirigen. Se halló que el valor de mercado del capital y la deuda decrece cuando se cambia al director, así como el flujo de efectivo esperado es mayor en las empresas que no cambian a sus directores. Mantener al director por el contrario tendría una correlación positiva con el tamaño de la firma, el apalancamiento, las compensaciones salariales y el flujo de efectivo (Berkovitch, Israel, \& Spiegel, 2000).

Existen dos teorías principales que buscan abordar el tema de la estructura de capital desde perspectivas distintas y predicciones diferentes. La primera es la Teoría del Equilibrio Estático (Trade-off Theory of Capital Structure), la cual hace énfasis en los impuestos, las dificultades financieras y el conflicto de intereses; y la segunda, la Teoría de la Jerarquía Financiera (Pecking Order Theory) que enfatiza la asimetría de información entre directores, accionistas $\mathrm{y}$ acreedores, $\mathrm{y}$ en la independencia financiera como determinantes de la estructura de capital.

El objetivo de esta investigación es descubrir los determinantes de la estructura de capital de 304 empresas del sector manufacturero de Guayaquil, durante el periodo 2012-2016 y qué teoría condensa mejor las decisiones de financiación de las empresas. La importancia del mismo, reside en verificar los determinantes del endeudamiento en empresas de un país en vías de desarrollo y comparar los resultados con las predicciones de las teorías sobre el Equilibrio Estático y sobre la Jerarquía Financiera. Es decir, comprobar las predicciones de estas dos teorías, para generar evidencia que permita determinar el poder explicativo de las mismas en empresas en países latinoamericanas en vías de desarrollo. En este sentido, también, se espera que los resultados del presente trabajo aporten al conocimiento general sobre la conducta financiera de empresas de países en vías de desarrollo y mediante los resultados obtenidos, a las teorías de la estructura de capital.

\section{Marco teórico}

\section{Estructura de capital: teorías}

El trabajo de Modigliani \& Miller fue el primero en exponer qué existe detrás de las decisiones de la estructura de capital de las corporaciones. En su trabajo, estos argumentaron que en un mercado perfecto el valor de las empresas no está influenciado por las decisiones de la estructura de capital. Esto implicaba que la existencia de impuestos, costos de agencia, costos de capital, costos de bancarrota y la asimetría de información no tenían relevancia alguna en la mezcla de deuda-capital y que más bien su valor dependía de sus activos. 
Sin embargo, existían ciertos problemas en su teoría: esta no integraba situaciones de la vida real, es decir, no incluía las imperfecciones de mercado. Años después, ambos autores publicaron un artículo indicando que habían incurrido en un error al no adaptar sus teorías a las condiciones reales (Modigliani \& Miller, 1963). Ambos reconocieron las ventajas impositivas particulares del uso de la deuda, pues contraer deuda genera intereses que son deducibles del pago de impuestos. En otras palabras, afirmaron que los impuestos sí eran determinantes de la estructura de capital, contrario a su previo trabajo de investigación. Sin embargo, advirtieron que, a pesar de las ventajas del endeudamiento, las empresas no deberían contraer el máximo de deuda potencialmente garantizada por sus activos, puesto que principalmente esto pudiera incrementar las probabilidades de caer en bancarrota.

Inicialmente en el presente trabajo se mencionaron dos teorías: La Teoría del Equilibrio Estático (TEE) y la Teoría de la Jerarquía Financiera (TJF). Cada teoría presenta predicciones diferentes, es decir, efectos distintos de los determinantes en la mezcla de deuda-capital.

Existen estudios que han indicado que el costo esperado de bancarrota sí tiene influencia en la estructura de capital (Warner, 1977). Warner (1977), tras analizar la estructura de capital de empresas de ferrocarriles llegó a la conclusión de que los costos de bancarrota tienen una relación inversa con el valor de mercado de la empresa, es decir, los costos bajan cuando el valor de la empresa incrementa, y podrían llegar a ser del $1 \%$ del valor de la empresa. Modigliani \& Miller (1963) reconocieron las ventajas impositivas particulares del uso de la deuda, pues contraer deuda genera intereses que son deducibles del pago de impuestos. En otras palabras, descubrieron que los impuestos sí eran determinantes de la estructura de capital. Sin embargo, advirtieron que, a pesar de las ventajas del endeudamiento, las empresas no debían contraer el máximo de deuda potencialmente garantizada por sus activos, puesto que este hecho incrementaría principalmente las probabilidades de bancarrota.

De acuerdo a la Teoría del Equilibrio Estático (TEE), la estructura de capital de las empresas viene determinado por la evaluación de costo-beneficio respecto al uso de deuda para financiar las operaciones (Serrasqueiro \& Caetano, 2015). Puntualmente, de acuerdo a la TEE, las decisiones de financiamiento dependen de tres aspectos: a) impuestos, b) costos de bancarrota y c) costos de agencia (Acaravci, 2016). Esta teoría menciona que las empresas equilibran las ventajas impositivas obtenidas por la deuda, contra las probabilidades de caer en bancarrota (Warner, 1977). Es decir, trata de predecir el nivel de deuda óptimo que deben fijar los administradores a fin de minimizar los costos de bancarrota y maximizar las ventajas impositivas (Serrasqueiro, Matias, \& Salsa, 2016; DeAngelo \& Masulis, 1980).

Este nivel de deuda objetivo y óptimo estaría relacionado inversamente con los costos esperados de bancarrota y con la cantidad de escudos fiscales no generados por deuda (Bradley, Jarrell, \& Kim, 1984). Estos últimos fueron incluidos en el modelo de Miller (1977) por (DeAngelo \& Masulis, 1980). Otros estudios han demostrado que las empresas con cero o poca deuda tienen diferenciales de impuestos más altos, indicando la nula necesidad de escudos fiscales (Kolay, Schallheim, \& Wells, 2013).

Por otra parte, esta teoría indica que mediante el endeudamiento los problemas de agencia tienden a reducirse, ya que, el endeudamiento serviría como un mecanismo disciplinario para los directores gracias a que la carga de la deuda, prevendría el uso ineficiente del efectivo. (Fama \& French, 2002). El motivo por el cual el endeudamiento funciona como un mecanismo de control de los directores reside en que el repago de la deuda exige salidas constantes y periódicas de efectivo en forma de capital e intereses, de tal forma que limita el efectivo 
disponible únicamente para desembolsos estrictamente necesarios. Las limitaciones de efectivo, en ciertos contextos, podrían evitar que el director se desvíe de los objetivos de los accionistas y su compromiso con ellos. Los administradores y directores por lo general están motivados por lograr sus propios objetivos, los mismos que podrían diferir con la maximización del valor de la firma para los accionistas (Eriotis, Vasiliou, \& Ventoura-Neokosmidi, 2007). El apalancamiento financiero reduciría los conflictos entre los accionistas y el director, pero los incrementaría entre los accionistas y acreedores (Frank \& Goyal, 2009; Myers, 1977) dado que el derecho al flujo de efectivo de la empresa estaría condicionado por la combinación deudacapital vigente en el momento.

Respecto a la Teoría de la Jerarquía Financiera (TJF) argumenta que las corporaciones no deberían perseguir un nivel de deuda óptimo (Myers, 1984; Myers \& Majluf, 1984). Es decir, que le otorga poca importancia al financiamiento externo y más bien prioriza financiarse por medio de recursos propios. Adicionalmente, señala que las decisiones de financiamiento siguen un orden jerárquico que se rige por el costo implícito de acceso al capital y la disponibilidad del mismo (Myers, 1984). En consecuencia, las empresas preferirían utilizar los fondos propios retenidos, seguido de los aportes de capital por socios existentes o nuevos, y finalmente la deuda.

El uso de la deuda estaría condicionado por la existencia de fondos propios, es decir, se usaría la deuda cuando los fondos propios se agoten. Esta teoría se enfoca en la relación de la asimetría de la información y las opciones de financiamiento. Los directores son quienes tienen acceso a información "privilegiada" respecto al futuro de la empresa (Chen \& Chen, 2011), de manera que son ellos quienes tienen una visión más clara de los riesgos que contraería la empresa bajo cualquiera de los escenarios, y cuáles serían los efectos en el rendimiento de los accionistas. Este hecho evidentemente influye en las decisiones financieras, principalmente porque los accionistas esperan que el valor de la empresa se maximice. Por supuesto que bajo esta hipótesis no se está tomando en cuenta el riesgo del o los proyectos en donde se coloquen los fondos, pero desde un punto de vista de maximización de valor de la firma financiarse mediante fondos propios resulta ser la mejor opción. Por otro lado, si los inversionistas creen que la empresa está infravalorada, estarán reacios a permitir que el director emita nuevo capital, de modo que elegirán el uso de deuda (Ferrer \& Tanaka, 2009) para prevenir la dilución de participación.

La asimetría de información es evidente entre los directores y acreedores cuando las condiciones crediticias entre acreedor y deudor dependen del valor de mercado del colateral. Si la empresa tiene activos colaterales de gran valor, el acreedor estará más dispuesto a solicitar menos información acerca de la condición de la empresa porque el valor del colateral es "suficiente" (Leland, 1998), y asume que el riesgo de bancarrota es menor, algo que beneficiaría tanto al acreedor como al deudor. Entonces, bajo este escenario, el costo de capital se reduce. Pero, paralelamente, existirían conflictos entre accionistas y acreedores debido a la transferencia de riqueza del accionistas al acreedor (Jorgensen \& Terra, 2003).

\section{Evidencia empírica en América Latina}

Padilla Ospina, Rivera Godoy, \& Ospina Holguín (2015) realizaron un estudio de empresas MiPyME en Colombia, en el periodo 2007-2011, en donde analizaron 309 empresas buscando determinar la relación e incidencia de seis variables independientes (edad de la empresa, tangibilidad, riesgo del negocio, rentabilidad, roe y tamaño) sobre el endeudamiento empresarial, sea a corto o largo plazo. Utilizaron el análisis de datos de panel, acompañado del modelo de efectos fijos. Hallaron una relación positiva y significativa entre la deuda y la 
tangilidad; así mismo con la rentabilidad y el tamaño de la empresa.

Hernández \& Ríos (2012) analizaron los determinantes de la estructura de capital de 14 empresas mexicanas pertenecientes a la industria alimenticia. El periodo de estudio fue desde el 2000 hasta el 2009. Se analizaron las siguientes variables independientes: tamaño de la empresa, rentabilidad, riesgo de negocio y tangibilidad. La variable dependiente fue el nivel de endeudamiento medido como deuda total/capital total. Sus hallazgos fueron "que los activos tangibles son la principal variable que estas empresas consideran para definir sus decisiones de financiamiento".

Tenjo, López \& Zamudio (2006) realizaron un estudio en Colombia, en donde buscaron estimar la incidencia de cinco variables independientes (rentabilidad, ventas [usado como proxy de tamaño], tangibilidad de activos, sector y característica idiosincrásica) sobre el ratio de endeudamiento total, medido como el total de la deuda sobre el total de activos. Un aspecto bastante importante del estudió fue que estos lo aislaron dentro de un contexto de crisis que existió en el país en los últimos años. El periodo de estudio comprendió los años 1996 a 2002 e involucró aproximadamente a 7,326 empresas por año. Utilizaron un método llamado Regresión por Cuantiles que "utiliza la distribución completa del endeudamiento de las firmas, condicional a un conjunto de variables explicativas". En términos generales hallaron una relación negativa entre el nivel de deuda y la rentabilidad; relación positiva entre el tamaño de la empresa y el nivel de deuda; y una relación negativa entre la tangibilidad y el nivel de deuda. Los resultados tuvieron concordancia con las predicciones de la Teoría de la Jerarquía Financiera.

Paredes, Ángeles, \& Flores (2016) estudiaron 14 empresas mineras públicas -que cotizan en bolsa- a lo largo de cinco países latinos: México, Chile, Colombia, Perú y Brasil. El periodo de estudio fue desde 2004 hasta 2004 en intervalos trimestrales. Ellos consideraron cinco variables: ratio de deuda total (variable dependiente), tangibilidad de activos, tamaño de la empresa (logaritmo natural de las ventas), oportunidades de crecimiento y la rentabilidad. Utilizaron una estructura de datos de panel junto al modelo de efectos fijos. Se encontró que la tangibilidad, el tamaño y la rentabilidad influyen negativa y significativamente en el nivel de deuda de las empresas mineras. Tales resultados tuvieron más coherencia con la Teoría de la Jerarquía Financiera.

Franco, López, \& Muñoz (2010) buscaron establecer "los factores determinantes del endeudamiento de las empresas manufactureras nacionales [...]" en empresas uruguayas. Optaron por utilizar la regresión lineal múltiple como herramienta para trabajar con datos transversales en un periodo de tres años: $2004-5$ y -6 . El tamaño de la muestra fue de 38 empresas para los dos primeros años, y 30 para el último año. Tomaron en cuenta 9 variables independientes y 1 dependiente. Las variables independientes fueron: el tamaño, la tangibilidad, rentabilidad, beneficio impositivo, orientación exportadora, fondos propios, flujo de fondos, impacto del sector y la edad de la empresa. La variable dependiente fue el ratio de deuda total. Solo la variable "flujo de fondos" tuvo una relación significativa y negativa con el endeudamiento.

\section{Datos y Metodología}

Para alcanzar los objetivos propuestos, planteamos un estudio exploratorio, empírico y cuantitativo sobre las pequeñas y medianas empresas manufactureras de Guayaquil, durante el periodo 2012-2016. La población objeto de estudio se ha obtenido de las empresas asociadas a 
la Cámara de la Pequeña Industria del Guayas $(\mathrm{CAPIG})^{1}$. Para realizar el estudio, se tomaron las 304 empresas de las que se disponía de información suficiente de los cinco años objeto de estudio.

La CAPIG agrupa a sus asociados en 25 sectores distintos. Dentro de la muestra final, existen únicamente 5 sectores que agrupan más del 50\% de las empresas: Químico (18.42\%), Alimentos y Bebidas (10.20\%), Metalmecánico (8.88\%), Servicios (7.57\%) y Plásticos y Caucho (6.91\%). En el 2016, las ventas de las empresas muestreadas llegaron a los $\$ 1.38$ billones de dólares (1.43\% del PIB) en conjunto, mientras que el PIB nominal ecuatoriano del mismo año, de acuerdo a datos del Banco Mundial, fue de $\$ 97.8$ billones de dólares.

En este estudio se han tomado en cuenta solamente las empresas constituidas de acuerdo a la normativa de la Superintendencia de Compañías y Valores, eliminando las individuales al no existir ningún tipo de información pública acerca de su situación financiera. Generalmente, investigaciones similares, se han centrado en analizar las empresas que cotizan en bolsa. Sin embargo, el mercado accionarial ecuatoriano no es representativo en comparación al de otras economías, por lo que el estudio se ha centrado en las PyME al ser de mayor utilidad, desde un punto de vista comparativo con el de otros países del entorno.

Dado que el intervalo de tiempo cubre desde el 2012 hasta el 2016, el primer paso fue eliminar las empresas con información incompleta y con menos años de información financiera que los que requería el estudio. A continuación, se recolectaron los estados financieros y los balances de las empresas disponibles, de donde se extrajeron todos los datos necesarios para el estudio. Todos los datos se obtuvieron a través de la página web de la Superintendencia de Compañías, Valores y Seguros de Ecuador ${ }^{2}$ y se trabajó con valores al cierre de año. Se usó Microsoft Excel para ordenar y adaptar los datos en forma de datos de panel, para posteriormente exportarlos a la herramienta estadística Stata v. $14^{3}$, con la que se generaron los modelos estadísticos para el estudio junto a las correspondientes pruebas estadísticas.

\section{Las variables determinantes de la estructura de capital}

Las variables analizadas y determinantes del endeudamiento para las empresas de la muestra son las siguientes:

Rentabilidad: La Teoría del Equilibrio Estático predice una relación positiva entre el endeudamiento de la empresa y la rentabilidad. Lo que esta teoría asume es que la empresa al ser altamente rentable puede incurrir en más deuda, lo que se traduce en un creciente pago de intereses que se deducen de los impuestos (Kouki \& Said, 2012; Padilla Ospina, Rivera Godoy, \& Ospina Holguin, 2015).

Por otro lado, la Teoría de la Jerarquía Financiera predice una relación negativa entre el endeudamiento y la rentabilidad. Esta asume que las empresas prefieren utilizar recursos propios antes que endeudarse, por lo tanto, mientras más rentable sea la empresa, hará menor uso de deuda como recurso financiero. Muchos estudios han demostrado mediante experimentos empíricos la validez de la TJF (Serrasqueiro, Matias, \& Salsa, 2016; Saeedi \& Mahmoodi, 2009; Acaravci, 2016; Serrasqueiro \& Caetano, 2015; Booth, Aivazian, Demirguc-Kunt, \& Maksimovic, 2001; Tenjo, López, \& Zamudio, 2006; Paredes, Ángeles, \& Flores, 2016). Este se medirá mediante la siguiente fórmula:

\footnotetext{
${ }^{1}$ http://www.capig.org.ec/. Esta institución está constituida por empresas que en su mayoría pertenecen a la categoría PyME. Cuenta con 521 asociados, de los cuales 433 son empresas constituidas y el resto son personas naturales.

${ }^{2}$ Puede consultarse en; www.supercias.gob.ec

${ }^{3}$ https://www.stata.com/stata14/
} 


$$
R E N T=\frac{\text { Ganancia antes de impuesto }}{\text { Activo_total }}
$$

Tamaño: La Teoría del Equilibrio Estático predice una relación positiva entre el tamaño y el endeudamiento. Esta relación existiría a partir del supuesto de que una empresa de gran tamaño está mucho más diversificada que una de menor tamaño. La diversificación, per se, reduciría 1) el riesgo de bancarrota y 2) la volatilidad de los ingresos. Por otro lado, al ser grandes empresas tienen mayor poder de negociación con los acreedores. Varios estudios han demostrado esta relación positiva (Acedo, Alútiz, \& Ruiz, 2012; Kouki \& Said, 2012; Eriotis, Vasiliou, \& Ventoura-Neokosmidi, 2007; Serrasqueiro \& Caetano, 2015; Padilla Ospina, Rivera Godoy, \& Ospina Holguin, 2015; Tenjo, López, \& Zamudio, 2006), mientras que otros han demostrado que no existe relación estadísticamente significativa (Tanaka, 2008; Hernández \& Ríos, 2012).

Por otra parte, la TJF predice una relación negativa. El argumento principal es que las grandes empresas tienen mejor y más fácil acceso a mercados de capital, dejando de lado la deuda. Estas están más dispuestas a entregar más y mejor información a los posibles inversionistas, en comparación con empresas pequeñas. Otro hecho importante es que al ser de gran tamaño poseen mayores ganancias retenidas con lo cual financiarse (Saeedi \& Mahmoodi, 2009; Acaravci, 2016; Sultan \& Adam, 2015; Paredes, Ángeles, \& Flores, 2016). Esta se medirá mediante la siguiente fórmula:

$$
T A M=\ln (\text { ingresos })
$$

Escudos fiscales no generados por deuda $\left(E F N D^{4}\right)$ : la TEE predice una relación negativa entre el endeudamiento y los EFND. A pesar de las ventajas fiscales que ofrece el uso de deuda, DeAngelo \& Masulis (1980) sugieren que los EFND pueden ser sustitutos casi perfectos de la deuda para reducir el pago de impuestos. Hay una discrepancia respecto al efecto de los EFND en la estructura de capital: varias investigaciones sugieren un efecto positivo (Serrasqueiro, Matias, \& Salsa, 2016; Serrasqueiro \& Caetano, 2015), así como negativo y en otros casos ningún efecto en absoluto (Saeedi \& Mahmoodi, 2009; Acaravci, 2016; Titman \& Wessels, 1988). Este se medirá mediante la siguiente fórmula:

$$
E F N D=\frac{\text { Gasto_Depreciación }}{\text { Activo_total }}
$$

Tangibilidad: la TEE predice un efecto positivo de la tangibilidad de los activos en relación al ratio de endeudamiento, porque el alto valor de los activos es más apreciado por los acreedores y puede ser usado como colateral para contraer más deuda. La literatura sugiere dos motivos: 1) los costos de agencia entre deudor y acreedor se reduce cuando los activos tienen alto valor de mercado y 2) un alto valor del colateral reduce las probabilidades de bancarrota, y aunque esto sucediera, el acreedor incurriría en menor riesgo de no recobrar la deuda.

\footnotetext{
${ }^{4}$ La depreciación, amortización, créditos fiscales por inversión.
} 
Contrariamente, la TJF predice una relación negativa entre la tangibilidad y el nivel de deuda, debido a que esta se enfoca en el uso de recursos internos para financiarse, así que al seguir esta lógica se descarta la deuda como una opción. Los resultados de varios estudios son mixtos: se han demostrado efectos negativos (Serrasqueiro, Matias, \& Salsa, 2016; Kouki \& Said, 2012; Saeedi \& Mahmoodi, 2009; Titman \& Wessels, 1988; Booth, Aivazian, DemirgucKunt, \& Maksimovic, 2001) y positivos (Huang \& Song, 2006; Hernández \& Ríos, 2012; Padilla Ospina, Rivera Godoy, \& Ospina Holguin, 2015). Este se medirá mediante la siguiente fórmula:

$$
T A N G=\frac{A c t \_F i j o \_N e t o}{A c t i v o \_t o t a l}
$$

Oportunidades de crecimiento: la TEE predice una relación negativa entre el endeudamiento y oportunidades de crecimiento. De acuerdo a la TEE, las empresas con un alto crecimiento esperado tienen menos activos tangibles que puedan servir como colateral de deuda, por ello prefieren -y requieren- financiarse por medio de recursos propios o capital externo. Consecuentemente, su nivel de deuda tiende a ser muy reducido: las grandes oportunidades de inversión agravan los problemas de agencia entre accionistas/directores y acreedores, al igual que los costos de bancarrota (Myers, 1984), dificultando el financiamiento mediante la deuda. Varias investigaciones han apoyado la hipótesis de la TEE (Serrasqueiro, Matias, \& Salsa, 2016; Eriotis, Vasiliou, \& Ventoura-Neokosmidi, 2007). En cambio, Mou (2011), Serrasqueiro \& Caetano (2015) y Titman \& Wessels (1988) demostraron ninguna relación significativa entre ambas variables.

En contraste, la TJF predice una relación positiva entre las variables. Las empresas con grandes oportunidades de crecimiento requieren mayor cantidad de fondos para adquisición de activos. Cuando los recursos propios se agotan, las empresas prefieren deuda en lugar de capital para financiarse, porque que grandes inversiones por lo general conllevan mayores riesgos. Varios estudios empíricos han apoyado esta hipótesis (Saeedi \& Mahmoodi, 2009; Kouki \& Said, 2012; Acaravci, 2016). Esta se medirá mediante la siguiente fórmula:

$$
O C=\frac{\Delta \text { Ingresos }_{t-1}}{\Delta \text { Activo_total }_{t-1}}
$$

Liquidez: la TEE predice una relación positiva argumentando que las empresas con altos ratios de liquidez tiene mayor capacidad de pago de obligaciones a tiempo, de modo que las empresas con alta liquidez deberían solicitar más deuda (Serrasqueiro, Matias, \& Salsa, 2016).

Ahora bien, la TJF sugiere una relación negativa entre la liquidez y el endeudamiento. La lógica detrás de esto sugiere que las empresas primero [y prefieren] usan las ganancias retenidas antes que emitir instrumentos para recoger capital. Estudios empíricos previos apoyan este supuesto (Serrasqueiro, Matias, \& Salsa, 2016; Saeedi \& Mahmoodi, 2009; Eriotis, Vasiliou, \& Ventoura-Neokosmidi, 2007). Esta se medirá mediante la siguiente fórmula:

$$
\text { Liq }=\frac{\text { Activo_cte }}{\text { Pasivo_cte }}
$$


Tanto las variables y la metodología de esta investigación han sido utilizadas y contrastadas en estudios previos como los de (Booth, Aivazian, Demirguc-Kunt, \& Maksimovic, 2001; Titman \& Wessels, 1988; Jorgensen \& Terra, 2003; Frank \& Goyal, 2009; Rajan \& Zingales, 1995; Sheikh \& Wang, 2011). Esto permite realizar comparaciones con otros estudios realizados en países e industrias diferentes, puesto que existe una falta de información respecto a los determinantes de la estructura de capital en países subdesarrollados y en vías de desarrollo, principalmente en Latinoamérica y zonas de Asia.

Por tanto, la variable dependiente de la investigación es "el ratio de endeudamiento total (RD)", medido como deuda total / activo total. Dicha variable se explicaría por las siguientes variables:

Tabla 1

Lista de variables independientes/explicativas.

\begin{tabular}{ll}
\hline Variables & Descripción \\
\hline RENT & Rentabilidad \\
TAM & Tamaño \\
EFND & Escudos fiscales no generados por deuda \\
TANG & Tangibilidad \\
LIQ & Liquidez \\
OC & Oportunidades de crecimiento \\
\hline
\end{tabular}

Fuente: Elaboración propia.

Por tanto, en esta investigación, según los objetivos planteados y a partir de las variables explicativas del endeudamiento, se formulan las siguientes hipótesis:

Lista de hipótesis a contrastar.

\begin{tabular}{ll}
\hline H1: & Existe una relación positiva entre la rentabilidad y el endeudamiento. \\
\hline H2: & Existe una relación negativa entre la rentabilidad y el endeudamiento. \\
H3: & Existe una relación positiva entre el tamaño y el endeudamiento. \\
H4: & Existe una relación negativa entre el tamaño y el endeudamiento. \\
H5: & Existe una relación positiva entre el endeudamiento y los escudos fiscales no generados por deuda. \\
H6: & Existe una relación positiva entre la tangibilidad y el endeudamiento. \\
H7: & Existe una relación negativa entre la tangibilidad y el endeudamiento. \\
H8: & Existe una relación negativa entre el endeudamiento y las oportunidades de crecimiento. \\
H9: & Existe una relación positiva entre las oportunidades de crecimiento y el endeudamiento. \\
H10: & Existe una relación negativa entre la liquidez y el endeudamiento. \\
H11: & Existe una relación positiva entre la liquidez y el endeudamiento. \\
\hline
\end{tabular}

Fuente: Elaboración propia.

Para el contraste de dichas hipótesis se utilizaron tres modelos distintos: a) Modelo de Mínimos Cuadrados Ordinarios (MCO), b) Modelo de Efectos Fijos y c) Modelo de Efectos Aleatorios, para estimar el efecto de cada variable explicativa en la variable dependiente, ratio de deuda, al igual la significancia estadística de cada una de ellas en cada modelo. Se consideró un nivel de error de 5\% (0.05), junto a un nivel de confianza de 95\%. La ecuación de los tres modelos, el MCO, efectos fijos y aleatorios, se presentan a continuación en el mismo orden: 


$$
\begin{gathered}
R D_{i t}=\beta_{0}+\beta_{1} R E N T_{i t}+\beta_{2} T_{A M}+\beta_{3} E F N D_{i t}+\beta_{4} T A N G_{i t}+\beta_{5} L I Q_{i t} \\
+\beta_{6} O C_{i t}+\varepsilon_{i t} \\
R D_{i t}=\beta_{0 i}+\beta_{1} R E N T_{i t}+\beta_{2} T_{A M}+\beta_{3} E F N D_{i t}+\beta_{4} T A N G_{i t}+\beta_{5} L I Q_{i t} \\
+\beta_{6} O C_{i t}+\mu_{i t} \\
R D_{i t}=\beta_{0}+\beta_{1} R E N T_{i t}+\beta_{2} T A M_{i t}+\beta_{3} E F N D_{i t}+\beta_{4} T A N G_{i t}+\beta_{5} L I Q_{i t} \\
+\beta_{6} O C_{i t}+\mu_{i t}+\varepsilon_{i t}
\end{gathered}
$$

Donde $i$ es la empresa, y $t$ es el año, $\varepsilon_{i t}$ es el error estocástico de la empresa $i$ en el tiempo t, $\mu_{\mathrm{it}}$ es el término de error de la empresa $i$ en el tiempo $t$.

El principal problema de utilizar el MCO para analizar datos de panel radica en que esta técnica no toma en cuenta los efectos no observables propios de cada empresa; efectos que podrían variar en el tiempo. El mecanismo de análisis implica agrupar todas las observaciones y asumir que cada dato, independientemente del periodo al que pertenezca, es una observación que no toma en cuenta a cada individuo en sí. Esto podría causar sesgo e inconsistencia en las estimaciones. Diversos estudios sugieren no utilizar los MCO para abordar esta situación con esa herramienta (Serrasqueiro, Matias, \& Salsa, 2016; Labra \& Torrecillas, 2014; Paredes, Ángeles, \& Flores, 2016).

Dicho esto, se ejecutaron los modelos de efectos fijos y efectos aleatorios, y posteriormente se realizó la prueba de Hausman para determinar cuál de los dos modelos se adapta mejor.

De acuerdo a la literatura, existe la posibilidad de que se correlacionen variables inobservadas con las variables del modelo. Decidir cuál modelo usar depende, entonces, de la existencia de esa correlación: si no existe correlación entre los efectos individuales inobservados de las empresas con las variables independientes lo recomendado es abordar el problema mediante el modelo de efectos aleatorios. De lo contrario, se recomienda utilizar el modelo de efectos fijos. La ventaja de utilizar el modelo de efectos fijos es que sí toma la individualidad de las empresas a lo largo del tiempo, dejando que el intercepto varíe para cada una (Sheikh \& Wang, 2011).

$\left.x^{2}\right)$. Este test compara los coeficientes de ambos modelos para determinar si las diferencias presentes son significativas, o no. La hipótesis nula (Ho) del test indica que no existe diferencia entre los coeficientes del modelo, es decir, no existe correlación entre los regresores y los efectos individuales. El criterio de rechazo es $x^{2}>0.05$ se rechaza la hipótesis nula. En consecuencia, se decide que el modelo más eficiente es el de efectos aleatorios. Caso contrario, se elige el modelo de efectos fijos (Labra \& Torrecillas, 2014).

Se revisó la presencia de multicolinealidad entre las variables, es decir, si existe una relación lineal perfecta entre una o varias de las variables explicativas. El problema de la multicolinealidad, según la teoría, es que si esta es perfecta los coeficientes de regresión de cada variable es indeterminado, y sus errores estándares son infinitos. Como medida de prueba se usó el Factor Inflacionario de la Varianza (FIV). Un FIV bajo, por ejemplo, 1, significa baja correlación; en contraste, un FIV de entre 1 y 5, significa correlación moderada, mientras que un valor mayor que 5 implica una alta correlación. 


\section{Resultados y discusión}

Esta sección muestra los resultados del modelo de efectos fijos, así como la estadística descriptiva que cada variable.

La figura 1 muestra el ratio de deuda total histórico desde el 2012 hasta el 2016 del total de las empresas ecuatorianas.

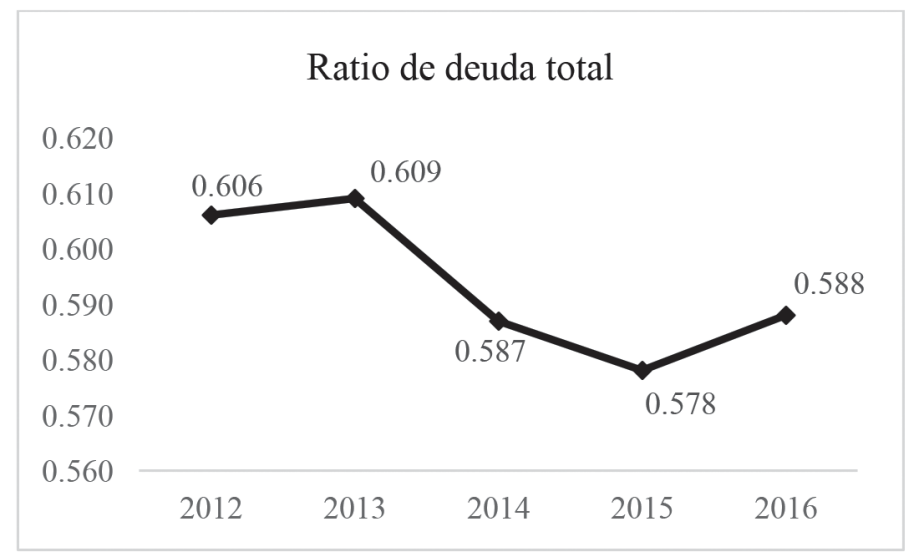

Figura 1: Ratio de deuda total de todas las empresas ecuatorianas.

Fuente: Superintendencia de Compañías, Valores y Seguros

Se observa que en todo el tiempo comprendido el ratio no tuvo una gran variación. En el 2012, por ejemplo, el $60.6 \%$ de los activos de las empresas estaba financiado por deuda, mientras que en el 2015 llegó a un nivel mínimo de 57.8\% y terminó repuntando hasta llegar a 58.8\%. Ecuador, al ser un país con una gran dependencia de los ingresos del petróleo, experimentó un fuerte golpe en su economía por la caída de los precios del crudo entre los años 2014-2015. De hecho, de acuerdo a reportes del Banco Central de Ecuador, el volumen de crédito otorgado por las entidades financieras tuvo una reducción drástica entre el periodo 2015-2016.

Los valores obtenidos de la estadística descriptiva se muestran en la siguiente tabla;

Tabla 2

Estadística descriptiva de las variables estudiadas.

\begin{tabular}{llllll}
\hline Variable & Obs. & Media & Desv. Est & Min & Max \\
\hline RD & 1,520 & 0.5749 & 0.2609 & 0.0001 & 1.9257 \\
RENT & 1,520 & 0.0871 & 0.1342 & -1.0697 & 0.9482 \\
TAM & 1,520 & 13.9179 & 1.7022 & 7.0244 & 19.3039 \\
EFND & 1,520 & 0.0386 & 0.0420 & 0.0001 & 0.5111 \\
TANG & 1,520 & 0.3482 & 0.2578 & 0.0000 & 0.9975 \\
LIQ & 1,520 & 4.6517 & 22.9721 & 0.0581 & 612.1120 \\
OC & 1,520 & 1.2610 & 209.1887 & -4926.3020 & 6050.3210 \\
\hline
\end{tabular}

Fuente: Elaboración propia.

El ratio de endeudamiento total (RD) indica que el 57.4\% de los activos totales de las empresas consideradas son financiados mediante la deuda, indistintamente de si esta es a largo o corto plazo. 
La rentabilidad, medida con el ROA, muestra una media de $8.7 \%$ junto a una desviación estándar de $13.4 \%$, indicando una gran variación de rendimientos sobre activos dentro de la muestra. La presencia de los escudos fiscales no generados por deuda solamente representa un $3.8 \%$ de los gastos en los balances, con una desviación estándar de 4.2\%. La tangibilidad, por su parte, indica que en promedio las empresas tienen un $34.8 \%$ de activos fijos netos en sus balances, con una desviación estándar de $25.8 \%$. Respecto a la liquidez, la media indica que los pasivos corrientes están cubiertos 4.7 veces por los pasivos corrientes, con una desviación estándar de 22.9. Finalmente, la variable OC (oportunidades de crecimiento) indica que, en promedio, por cada $1 \%$ de incremento en el activo total de las empresas, sus ventas incrementan 1.3 veces.

En la Tabla 3 se muestra la baja correlación de las variables entre sí y con el ratio de deuda.

Tabla 3

Matriz de coeficiente de correlación de Pearson.

\begin{tabular}{llllllll}
\hline \multicolumn{1}{c}{ RD } & RENT & TAM & EFND & TANG & LIQ & OC \\
\hline RD & 1.0000 & & & & & & \\
RENT & -0.3194 & 1.0000 & & & & & \\
TAM & 0.1640 & 0.1200 & 1.0000 & & & & \\
EFND & -0.0280 & 0.0723 & -0.1012 & 1.0000 & & & \\
TANG & -0.1125 & -0.1385 & -0.0123 & 0.3355 & 1.0000 & & \\
LIQ & -0.1925 & 0.0282 & -0.1923 & -0.0442 & -0.0375 & 1.0000 & \\
OC & 0.0122 & 0.0093 & 0.0272 & 0.0415 & 0.0514 & -0.1003 & 1.0000 \\
\hline
\end{tabular}

Fuente: Elaboración propia.

Se observa que la correlación entre los EFND y la TANG es la mayor positiva- de todas. Este hecho se explica debido a que mientras más activos fijos netos posea una empresa, mayor es el gasto por depreciación de esos activos, y por consecuencia la medida EFND incrementa simultáneamente con TANG. Por el contrario, las variables RD y RENT muestran una correlación negativa; esto hecho podría explicarse a debido a que un mayor nivel de deuda incrementa el pago de interés, reduciendo la utilidad antes de impuestos.

Tras los resultados de correlación, la Tabla 4 muestra el Factor Inflacionarios de la Varianza de cada variable. Notablemente, están en niveles bajos, 1.09 en promedio, indicando una baja correlación entre las variables. Gracias a esta prueba se puede afirmar que no existe un problema significativo de multicolinealidad.

Tabla 4

Factor inflacionario de la varianza.

\begin{tabular}{lll}
\hline Variable & FIV & 1/FIV \\
\hline EFND & 1.17 & 0.85673 \\
TANG & 1.17 & 0.85752 \\
TAM & 1.07 & 0.93117 \\
RENT & 1.06 & 0.94434 \\
LIQ & 1.06 & 0.94705 \\
OC & 1.01 & 0.98682 \\
\hline FIV prom & 1.09 & \\
\hline
\end{tabular}

A partir de los datos anteriores se ha testado el Modelo de Mínimos Cuadrados Ordinarios (MCO) y cuyos resultados se presentan en la siguiente Tabla. 
Tabla 5

Resultados del modelo MCO.

\begin{tabular}{lllll}
\hline $\mathrm{R}^{\wedge} 2$ & 0.1968 & $\mathrm{~F}$ & 61.7753 & \\
\hline $\mathrm{R}^{\wedge} 2$ ajustado & 0.1936 & Prob $>\mathrm{F}$ & 0.0000 & \\
Error típico & 0.2343 & $\mathrm{SSE}$ & 83.0256 & \\
Observaciones & 1520 & $\mathrm{SSR}$ & 20.3394 & \\
& & & & \\
Variables & Coef. & Err. Std & $\mathrm{t}$ & $\mathrm{P}>\mathrm{t}$ \\
$\mathrm{RENT}$ & -0.7181 & 0.0461 & -15.58 & 0.0000 \\
TAM & 0.0283 & 0.0037 & 7.73 & 0.0000 \\
EFND & 0.4672 & 0.1547 & 3.02 & 0.0030 \\
TANG & -0.1946 & 0.0252 & -7.73 & 0.0000 \\
LIQ & -0.0017 & 0.0003 & -6.35 & 0.0000 \\
OC & 0.0000 & 0.0000 & 0.1 & 0.9210 \\
Intercepción & 0.3013 & 0.0523 & 5.76 & 0.0000 \\
\hline
\end{tabular}

Fuente: Elaboración propia.

Previo a seleccionar el modelo que mejor explique las relaciones entre las variables se realizó la prueba de Hausman.

Tabla 6.

Prueba de Hausman para los modelos de efectos fijos y de efectos aleatorios.

\begin{tabular}{lllll}
\hline & E. Fijos & E. Aleatorios & Diferencia & \\
\cline { 2 - 4 } Variables & Coef & Coef & (ef-ea) & Err. Std. \\
\hline RENT & -0.50998 & -0.53835 & 0.02837 & 0.01278 \\
TAM & 0.03007 & 0.03005 & 0.00003 & 0.00831 \\
EFND & 0.46788 & 0.45842 & 0.00946 & 0.44423 \\
TANG & -0.10648 & -0.13130 & 0.02482 & 0.01614 \\
LIQ & -0.00061 & -0.00077 & 0.00017 & 0.00006 \\
OC & 0.00001 & 0.00001 & 0.00000 & 0.00000 \\
Const & 0.22253 & 0.23513 & & \\
Hausman & 17.690 & & & \\
p-value & 0.0034 & & & \\
\hline
\end{tabular}

Fuente: Elaboración propia.

La Tabla 6 indica un $p$-value $=0.0034$ que impide rechazar la hipótesis nula de que no existe correlación entre los efectos de los individuos y las variables explicativas. Por lo tanto, se decidió que el modelo adecuado era el de efectos fijos.

De acuerdo a los resultados del Modelo de Efectos Fijos, Tabla7, la rentabilidad, el tamaño, los escudos fiscales no generados por deuda, la tangibilidad de los activos y la liquidez son altamente significativos bajo un 5\% de significación. Solamente la variable OC (oportunidades de crecimiento) parece ser altamente no significativo bajo el mismo nivel de significación. 
Tabla 7

Modelo de Efectos Fijos.

\begin{tabular}{llll}
\hline R^2 & 0.1830 & F & 38.09 \\
Corr (Ui_Xb) & 0.1334 & Prob $>$ F & 0.0000 \\
Observaciones & 1520 & Grupos & 304 \\
\hline Variables & Coef. & P > I t I & \\
\hline RENT & -0.5100 & 0.0000 & \\
TAM & 0.0301 & 0.0030 & \\
EFND & 0.4679 & 0.0010 & \\
TANG & -0.1065 & 0.0010 & \\
LIQ & -0.0006 & 0.0050 & \\
OC & 0.0000 & 0.4740 & \\
_cons & 0.2225 & 0.1190 & \\
\hline
\end{tabular}

Fuente: Elaboración propia.

El valor de $\mathrm{R}^{2}$ es de 0.1968 e indica que solamente el $19.6 \%$ de la variación del endeudamiento total (RD) está explicado por el efecto de las variables independientes.

En la Tabla 8 se muestran los resultados obtenidos del Modelo de Efectos Aleatorios, que mide el efecto de cada variable explicativa respecto a variable dependiente, ratio de deuda, con una significancia estadística de cada una de ellas en cada modelo. Se ha considerado un nivel de error de $5 \%$ (0.05), junto a un nivel de confianza de $95 \%$.

Los resultados empíricos sugieren que la rentabilidad tiene una relación negativa y altamente significativa sobre el nivel de deuda de las empresas. Este resultado tiene concordancia con lo esperado únicamente bajo la TJF, más no bajo la TEE. La TJF asume que las empresas prefieren utilizar recursos propios antes que endeudarse, por lo tanto, mientras más rentable sea la empresa, hará menor uso de deuda como recurso financiero. El resultado obtenido es similar al de otras investigaciones (Serrasqueiro, Matias, \& Salsa, 2016; Huang \& Song, 2006; Chen \& Chen, 2011; Saeedi \& Mahmoodi, 2009; Acaravci, 2016; Tanaka, 2008; Khrawish \& Khraiwesh, 2010; Booth, Aivazian, Demirguc-Kunt, \& Maksimovic, 2001; Eriotis, Frangouli, \& Ventoura-Neokosmides, 2011). Se acepta la hipótesis H2.

Tabla 8

Predicciones de cada teoría y los resultados del modelo de efectos fijos.

\begin{tabular}{lccc}
\hline Variable & \multicolumn{3}{c}{ Teoría } \\
\cline { 2 - 4 } & $\begin{array}{c}\text { TEE } \\
\text { Predicción }\end{array}$ & $\begin{array}{c}\text { TJF } \\
\text { Predicción }\end{array}$ & $\begin{array}{c}\text { Modelo } \\
\text { Signo }\end{array}$ \\
\hline Rentabilidad & + & - & $-*$ \\
Tamaño & + & - & $+^{*}$ \\
Escudos fiscales & - & $\mathrm{n} / \mathrm{a}$ & $+^{*}$ \\
Tangibilidad & + & - & $-*$ \\
Liquidez & + & - & $-*$ \\
Oportunidad de & - & + & + \\
crecimiento & & &
\end{tabular}

Fuente: Elaboración propia.

De acuerdo a la TEE, el tamaño tiene una relación positiva con el endeudamiento. Los resultados del modelo presentan una relación positiva y altamente significativa entre ambas variables, de esta manera se comprueba la hipótesis de la TEE respecto a esta relación. La explicación, de acuerdo a la teoría, es que mientras más grande sea la empresa esta tiene mayor 
diversificación y presenta un bajo riesgo de a) quiebra y 2) volatilidad de sus ingresos. Estas dos cualidades motivan a los directores a endeudarse a mayores niveles y relaja las exigencias por parte de los acreedores. Este resultado solamente apoya la predicción de la TEE, no de la TJF. El resultado de este estudio es similar al de otros autores (Acedo, Alútiz, \& Ruiz, 2012; Kouki \& Said, 2012; Eriotis, Vasiliou, \& Ventoura-Neokosmidi, 2007; Khrawish \& Khraiwesh, 2010; Serrasqueiro \& Caetano, 2015). Se acepta la hipótesis H3.

La TEE sugiere una relación negativa entre el nivel de deuda y los EFND. Los resultados del modelo arrojan una relación positiva y significativa entre las variables. La explicación de este suceso podría radicar en que las empresas utilizan sus activos fijos como colateral de deuda, lo que incrementaría los gastos en depreciación de activos al momento de la adquisición, y el nivel de deuda. Este resultado no apoya la predicción de la TEE. Otros estudios mostraron resultados similares al de este (Serrasqueiro, Matias, \& Salsa, 2016; Serrasqueiro \& Caetano, 2015; DeAngelo \& Masulis, 1980). Se acepta la hipótesis H5.

Tanto la TEE como la TJF predicen una relación positiva entre la tangibilidad de los activos y el nivel de deuda. Tal predicción estaría basada bajo el hecho de que las empresas ofrecen sus activos tangibles como colateral de deuda, así que mientras más activos tangibles tenga la empresa, mayor será su nivel de deuda. Los resultados del modelo sugieren lo contrario, es decir, ambas variables tienen una relación negativa, indicando que el nivel de deuda no debe, necesariamente, incrementarse ante la presencia de gran cantidad de activos tangibles. Este resultado es similar al de otras investigaciones (Serrasqueiro, Matias, \& Salsa, 2016; Kouki \& Said, 2012; Saeedi \& Mahmoodi, 2009; Acaravci, 2016; Booth, Aivazian, Demirguc-Kunt, \& Maksimovic, 2001). Un factor que podría causar esta relación negativa sería que los activos tangibles no tengan un alto valor de mercado, lo que desmotivaría a los acreedores a aceptarlos como colateral para deuda. Se acepta la hipótesis H7.

El modelo sugiere una relación negativa y significativa entre la liquidez y el ratio de deuda. Este resultado tiene concordancia con lo predicho en la TJF, pero no con la TEE. Este resultado es similar al de otras investigaciones (Serrasqueiro, Matias, \& Salsa, 2016; Saeedi \& Mahmoodi, 2009; Eriotis, Vasiliou, \& Ventoura-Neokosmidi, 2007). La lógica detrás de esta relación consiste en que las empresas prefieren usar las ganancias retenidas como forma de financiarse. Este resultado tiene gran consistencia con lo encontrado en un estudio de PyMEs en Guayaquil: casi la mitad de los directores de estas empresas indicaron que el principal problema al momento de financiarse es el costo del financiamiento, es decir, alta tasa de interés por el crédito (Moran, Jácome, \& Rodríguez, 2017). Se acepta la hipótesis H10.

Por último, la variable OC (oportunidades de crecimiento) no tiene ninguna importancia estadística en el modelo. Este resultado confirma que el crecimiento esperado de las empresas, considerado como un activo intangible, no puede ser usado como colateral de deuda, lo que implica que el ratio de deuda no se vea afectado.

\section{Conclusiones}

Este estudio empírico ha explorado los determinantes de la estructura de capital de 304 empresas guayaquileñas durante el periodo de 2012 - 2016 mediante una estructura de datos de panel y analizándola mediante un Modelo de Efectos Fijos.

Los resultados indican que existe una relación negativa entre la rentabilidad, la tangibilidad y la liquidez, respecto al endeudamiento corporativo. Contrariamente, el tamaño y los escudos 
fiscales no generados por deuda tienen una relación significativamente positiva con el endeudamiento. Solamente la variable "oportunidades de crecimiento" resulto no tener ninguna importancia estadística sobre el nivel de deuda.

La evidencia del poder explicativo de la Teoría del Equilibrio Estático en cuanto al tipo de financiamiento preferido por las empresas guayaquileñas es débil. Esta teoría sólo pudo predecir la relación de un determinante con el nivel de deuda con el tamaño de la empresa. Es decir, los resultados del modelo sugieren una relación significativa y positiva entre el tamaño y el nivel de deuda. Por otro lado, la Teoría de la Jerarquía Financiera fue capaz de predecir casi con exactitud la relación de cada factor y su relación con el nivel de deuda y con la elección de financiamiento. En consecuencia, predice mejor el comportamiento financiero de la muestra. Esta, predijo correctamente las relaciones de la rentabilidad, tangibilidad, liquidez y oportunidades de crecimiento (aunque esta última no tuvo significancia estadística), pero no la relación del tamaño de la empresa con el endeudamiento. Los resultados del modelo concuerdan con tres predicciones de esta teoría: una correlación negativa y significativa entre la rentabilidad, la liquidez, y el nivel de deuda; y positiva, pero no significativa entre las oportunidades de crecimiento y el nivel de deuda. Los resultados tienen coherencia con cuatro de las cinco predicciones de esta teoría.

La razón del por qué existe una relación negativa entre la rentabilidad, la tangibilidad y la liquidez, y una positiva entre el tamaño y los escudos fiscales no generados por deuda contra el nivel de deuda es la siguiente: las empresas prefieren utilizar recursos propios para financiarse, por lo tanto, mientras más rentable y líquida sea la empresa, menor deuda requiere. Respecto a la tangibilidad, las empresas con menores niveles de activos fijos tienen un mayor nivel de endeudamiento. Se presume que estas empresas 1) otorguen activos corrientes como garantía y/o 2) utilicen deuda a corto plazo como opción de financiamiento.

Respecto al tamaño y los escudos fiscales no generados por deuda, se presume que las empresas con mayor tamaño obtienen mayores créditos gracias al bajo riesgo de bancarrota e impago de las mismas, por el hecho de tener un negocio más diversificado que una empresa de menor tamaño. Por el lado de los escudos fiscales no generados por deuda, se presume que las empresas sí utilizan el endeudamiento para tomar ventaja de las deducciones fiscales por gasto por interés.

Con lo anterior se concluye que la empresa asociada a la CAPIG prefiere, definitivamente, financiarse por medios propios antes que contraer deuda con los bancos o emitir instrumentos de deuda. Estos resultados tienen concordancia con los obtenidos de Tenjo, López \& Zamudio (2006) de empresas colombianas, Paredes, Ángeles, \& Flores (2016) de empresas de cincos países latinos, Booth, Aivazian, Demirguc-Kunt, \& Maksimovic (2001) de empresas de diez países en vías de desarrollo y Acaravci (2016) de empresas turcas.

De acuerdo a un estudio realizado por la Universidad de Especialidades Espíritu Santo, presentado en el 2017, el 90\% de las empresas ecuatorianas existentes son familiares. Esto significa que es muy probable que tanto los accionistas como los directores estén emparentados entre sí, dejando poca cabida a inversionistas ajenos a la familia.

El costo de la deuda podría ser un aspecto importante a considerar para conocer mejor el por qué las empresas prefieren financiarse por medios propios antes que endeudarse. En Ecuador, la tasa de interés para créditos empresariales está en el rango de $7-10 \%^{5}$ anual en promedio;

\footnotetext{
${ }^{5}$ Banco Central del Ecuador, 2016-2017
} 
en Colombia, 8-12\% $\%^{6}$ mientras que en países como Chile y Perú se ubica en $5-7 \%^{7}$ y 3-5\% ${ }^{8}$, respectivamente.

Futuras líneas de investigaciones deberían integrar la tasa de interés histórica a los modelos para estimar el efecto que tienen estas en la elección de financiamiento por parte de las empresas. Además, sería enriquecedor conocer los determinantes de la estructura de capital por sector e industrias de la economía, agregando también una variable que segregue las empresas entre familiares o no familiares, para así se tener estimaciones más precisas sobre las preferencias de financiamiento corporativo con un amplio nivel de detalle.

\section{Referencias}

Acaravci, S. (2016). The Determinants of Capital Structure: Evidence from the Turkish Manufacturing Sector. International Journal of Economics and Financial Issues, 5(1), 158 - 171.

Acedo, M. A., Alútiz, A., \& Ruiz, F. (2012). Factores Determinantes de la Estructura de Capital de las Empresas Españolas. Tribuna de Economía,868, 155-172.

Berkovitch, E., Israel, R., \& Spiegel, Y. (2000). Managerial Compensation and Capital Structure. Journal of Economics \& Management Strategy, 9(4), 549-584. doi:https://doi.org/10.2139/ssrn.142951

Booth, L., Aivazian, V., Demirguc-Kunt, A., \& Maksimovic, V. (2001). Capital Structure in Developing Countries. The Journal of Finance,56(1) 87-130. doi:https://doi.org/10.1111/0022-1082.00320

Bradley, M., Jarrell, G., \& Kim, H. (1984). On the Existence of an Optimal Capital Structure: Theory and Evidence. The Journal of Finance, 39(3), 857-878. doi:https://doi.org/10.2307/2327950

Chen, L.-J., \& Chen, S.-Y. (2011). How the Pecking-Order Theory Explain Capital Structure. Journal of International Management Studies, 1-9.

DeAngelo, H., \& Masulis, R. (1980). Optimal Capital Structure Under Corporate and Personal Taxation. Journal of Financial Economic, 8(1), 3-29. doi:https://doi.org/10.1016/0304-405x(80)90019-7

Eriotis, N., Vasiliou, D., \& Ventoura-Neokosmidi, Z. (2007). How firm characteristics affect capital structure: an empirical study. Managerial Finance,33(5), 321-331. doi:https://doi.org/10.1108/03074350710739605

Fama, E., \& French, K. (2002). Testing Trade-off and Pecking Order predictions about dividend and debt. The Review of Financial Studies, 15 (1) 1-33. doi:https://doi.org/10.1093/rfs/15.1.1

Ferrer, M., \& Tanaka, Á. (2009). Las PyMES y las teorías modernas sobre estructura de capital. Compendium(22), 65-83.

Franco, G., López, L., \& Muñoz, G. (2010). Determinantes de la estructura de capital de las grandes empresas manufactureras en Uruguay. Econoquantun, 5, 4-25.

Frank, M., \& Goyal, V. (2009). Capital Structure Decisions: Which Factors Are Reliably Important? Financial Management, 38(1), 1-37. doi:http://dx.doi.org/10.1111/j.1755-053X.2009.01026.x

Hernández, G., \& Ríos, H. (2012). Determinantes de la estructura financiera en la industria manufacturera: la industria de alimentos. Análisis Económico,27 (65), 101-121.

Huang, S., \& Song, F. (2006). The Determinants of Capital Structure: Evidence from China. China economic review, 17 (1), 14-36. doi:https://dx.doi.org/10.2139/ssrn.320088

Jorgensen, J., \& Terra, P. (2003). Determinants of capital structure in Latinamerica: The role of firm-specific and macroeconomic factors. Annual Meeting of Multinational Finance Society.

Khrawish, H., \& Khraiwesh, A. (2010). The Determinants of the Capital Structure: Evidence from Jordanian Industrial Companies. Economics and Administration, 24(1), 173-196. doi:https://doi.org/10.4197/Eco.24-1.5 .

Kolay, M., Schallheim, J., \& Wells, K. (2013). A New Measure for Non-debt Tax Shields and the Impact on Debt Policy. University of Utah Working Paper.

\footnotetext{
${ }^{6}$ Banco de la República - Colombia, 2016-2017

${ }^{7}$ Banco Central de Chile, 2016-2017

${ }^{8}$ Banco Central de la Reserva del Perú, 2016-2017
} 
Kouki, M., \& Said, H. (2012). Capital Structure Determinants: New Evidence from French Panel Data. International Journal of Business and Management, 7(1). doi:http://dx.doi.org/10.5539/ijbm.v7n1p214

Labra, R., \& Torrecillas, C. (2014). Guía cero para datos de panel: un enfoque práctico. Madrid: UAM-Accenture.

Leland, H. (1998). Agency Costs, Risk Management, and Capital Structure. The Journal of Finance, 53 (4), 1213-1243. doi:http://dx.doi.org/10.1111/0022-1082.00051

Miller, M. (1977). Debt and Taxes. The Journal of Finance, 32 (2), 261-275. doi:http://dx.doi. org/10.1111/j.1540-6261.1977.tb03267.x

Modigliani, F., \& Miller, M. (1958). The Cost of Capital, Corporation Finance and the Theory of Investment. The American Economic Review, 48 (3), 261-297.

Modigliani, F., \& Miller, M. (1963). Corporate Income Taxes and the Cost of Capital: A Correction. The American Economic Review, 53(3), 433-443.

Moran, C., Jácome, D., \& Rodríguez, L. (2017). Emisiones sindicadas de obligaciones como alternativa de financiamiento para las PyME del sector metalmecánico en Guayaquil. Revista Ciencia UNEMI, 10(22), 11-19.

Mou, W. (2011). The Determinants of Capital Structure in Chinese Listed Companies. Masters thesis. Universiti Utara Malaysia.

Myers, S. (1977). Determinants of Corporate Borrowing. Journal of Finance, 5(2), 147-175. doi:https://doi. org/10.1016/0304-405X(77)90015-0

Myers, S. (1984). The Capital Structure Puzzle. The Journal of Finance, 39(3). doi:http://dx.doi. org/10.1111/j.1540-6261.1984.tb03646.x

Myers, S., \& Majluf, N. (1984). Corporate Financing and Investment Decisions when Firms Have Information The Investor do not Have. Journal of Financial Economics, 13(2). doi:https://doi.org/10.1016/0304-405X(84)90023-0

Padilla Ospina, A., Rivera Godoy, J., \& Ospina Holguin, J. (2015). Determinantes de la estructura de capital de las mipymes del sector real participantes del Premio Innova 2007-2011. Revista Finanzas y Política Económica, 7 (2), 359-380.

Paredes, A., Ángeles, G., \& Flores, M. (2016). Determinants of leverage in mining companies, empirical evidence for Latin American countries. Contaduría y Administración, 61 (1), 26-40.

Rajan, R., \& Zingales, L. (1995). What Do We Know about Capital Structure? Some evidence from international data. The Journal of Finance, 50 (5), 1421-1460.

Saeedi, A., \& Mahmoodi, I. (2009). The Determinants of Capital Structure: Evidence from an Emerging Market. Recent Advances in Business Administration, 17(1/2),13-18. doi:https://doi.org/10.1108/10569210710774730

Serrasqueiro, Z., \& Caetano, A. (2015). Trade-Off Theory versus Pecking Order Theory: capital structure decisions in a peripheral region of Portugal. Journal of Business Economics and Management, 16 (2), 445-466. doi:https://doi. org/10.3846/16111699.2012.744344

Serrasqueiro, Z., Matias, F., \& Salsa, L. (2016). Determinants of capital structure: New evidence from Portuguese small firms. Dos Algarves: A Multidisciplinary e-Journal, 28, 13-28. doi:http://dx.doi.org/10.18089/DAMeJ.2016.28.1.2

Sheikh, N., \& Wang, Z. (2011). Determinants of capital structure: an empirical study of firms in manufacturing industry of Pakistan. Managerial Finance, 37(2), 117-133. doi:https://doi.org/10.1108/03074351111103668

Sultan, A., \& Adam, M. (2015). The Effect of Capital Structure on Profitability: An Empirical Analysis of Listed Firms in Iraq. European Journal of Accounting, Auditing and Finance Research, 3(2), 61-78.

Tanaka, Á. (2008). Comportamiento de la Estructura Financiera en un Grupo de Empresas Españolas previo a la participación de capital de riesgo. Documentos de trabajo en finanzas de empresas(2).

Tenjo, F., López, E., \& Zamudio, N. (2006). Determinantes de la estructura de capital de las empresas colombianas: 1996-2002. Coyuntura Económica, XXXVI(1), 117-147.

Titman, S., \& Wessels, R. (1988). The Determinants of Capital Structure Choice. The Journal of Finance, 43(1), 1-19. doi:http://dx.doi.org/10.1111/j.1540-6261.1988.tb02585.x

Warner, J. (1977). Bankruptcy Costs: Some Evidence. The Journal of Finance, 32(2), 337-347. doi:http://dx.doi. org/10.1111/j.1540-6261.1977.tb03274.x 